\title{
Common and unusual urogenital Crohn's disease complications: spectrum of cross-sectional imaging findings
}

\author{
Massimo Tonolini, ${ }^{1}$ Chiara Villa, ${ }^{1}$ Alessandro Campari, ${ }^{2}$ Anna Ravelli, ${ }^{2}$ Roberto Bianco, ${ }^{1}$ \\ Gianpaolo Cornalba ${ }^{2}$
}

\author{
${ }^{1}$ Department of Radiology, "Luigi Sacco" University Hospital, Via G.B. Grassi 74, 20157 Milan, Italy \\ ${ }^{2}$ Department of Diagnostic and Interventional Radiology, San Paolo Hospital, Milan University, Via A. di Rudinì 8, 20142 Milan, \\ Italy
}

\begin{abstract}
Involvement of the urinary tract and genital organs is not uncommon in patients affected with Crohn's disease (CD). Occurring in both sexes, uro-gynecological complications are often clinically unsuspected because of the dominant intestinal or systemic symptoms. Knowledge of their manifestations and cross-sectional imaging appearances is necessary to recognize and report them, since correct medical or surgical treatment choice with appropriate specialist consultation allows to prevent further complications. Besides uncomplicated urinary tract infections that usually do not require imaging, urolithiasis and pyelonephritis represent the most commonly encountered urinary disorders: although very useful, use of computed tomography (CT) should be avoided whenever possible, to limit lifetime radiation exposure. Hydronephrosis due to ureteral inflammatory entrapment and enterovesical fistulization may result from penetrating $\mathrm{CD}$, and require precise imaging assessment with contrast-enhanced CT to ensure correct surgical planning. Representing the majority of genital complication, ano- and rectovaginal fistulas and abscesses frequently complicate perianal inflammatory CD and are comprehensively investigated with high-resolution perianal MRI acquired with phased-array coils, high-resolution T2-weighted sequences and intravenous contrast. Finally, rare gynecological manifestations including internal genital fistulas, vulvar and male genital involvement are discussed.
\end{abstract}

Correspondence to: Massimo Tonolini; email: mtonolini@sirm.org
Key words: Crohn's disease-PyelonephritisHydronephrosis-Vesical fistula-Vaginal fistula-Perianal inflammatory disease - Vulva - Computed tomography (CT) Magnetic resonance imaging (MRI)

Crohn's disease (CD) is a chronic inflammatory disorder of the alimentary tract, most usually involving the ileum and proximal colon and commonly associated with extraintestinal manifestations. Lifelong clinical follow-up of $\mathrm{CD}$ patients includes frequent endoscopic and crosssectional imaging studies $[1,2]$. Nowadays, contrast-enhanced multidetector computed tomography (CT) with multiplanar reformations is the mainstay diagnostic modality to investigate acute $\mathrm{CD}$ exacerbations, whereas volume-challenge $\mathrm{CT}$ or magnetic resonance imaging (MRI) techniques such as enteroclysis (with nasoenteric intubation) and peroral enterography studies allow precise assessment of intestinal disease extent, activity and postoperative recurrences. Furthermore, cross-sectional imaging studies allow identification of CD-related extraintestinal complications, most often occurring secondary to transmural penetration of inflammatory changes leading to fistulization into adjacent structures such as other intestinal loops, the abdominal wall and other viscera [2].

Pelvic MRI acquisition with phased-array coils, multiplanar high-resolution T2-weighted sequences and intravenous contrast currently represents the gold standard technique to image perianal fistulizing $\mathrm{CD}$, providing precise classification of disease topography, identification of abscess collections and confident followup after medical and surgical treatments [2-6]. 
Complications involving the urinary and genital organs are not uncommon in CD patients and are often clinically underestimated because of the dominant intestinal or systemic symptoms [7-10].

\section{Urologic complications}

Variably reported in literature (incidence range 4\%23\%), CD-related complications involving the urinary tract include infections and urolithiasis as the most common manifestations, whereas rare although serious occurrences associated with penetrating-type disease include ureteral obstruction and enterovesical fistulization.

Clinically, urinary complaints such as recurrent infections, fever, dysuria, pneumaturia, and fecaluria should be thoroughly investigated and clearly stated on radiology requests, to avoid missed or delayed diagnoses. Although cross-sectional imaging studies are focused on inflammatory bowel disease, radiologists should carefully search and report any possible urologic abnormality in CD patients $[7,8,11]$.

\section{Urolithiasis}

Occurring in up to $12 \%$ of $\mathrm{CD}$ patients overall, renal calculi are particularly frequent in patients with ileostomy or multiple bowel resections. Mechanisms include altered oxalate metabolism from malabsorption plus abnormal urinary $\mathrm{pH}$ due to intestinal fluid losses [12].

Widely available and very quickly performed on multidetector CT scanners, unenhanced CT is currently the mainstay modality to investigate urolithiasis. Complemented with helpful coronal reformations, unenhanced CT allows confident detection of even tiny hyperdense stones along the entire excreting system and identification of associated secondary signs of acute renal colic such as hydronephrosis, perinephric-periureteral edema, fascial thickening, and renal enlargement with absent white pyramid. Furthermore, unenhanced CT provides differentiation from other urinary and extraurinary conditions with similar clinical presentation, including acute gynecological conditions, pyelonephritis, appendicitis, diverticulitis, and vascular and musculoskeletal abnormalities [13].

Thus, CT may prove particularly appealing to emergency physicians and urologists because it provides simultaneous assessment of urolithiasis and of acute intestinal conditions in CD patients. Although recently low-dose acquisition protocols have been proposed, the key disadvantage of CT is represented by its non-negligible ionizing irradiation with associated theoretic risk of induced malignancy. Experiencing a lifelong disease course from a young age including frequent exacerbations, patients with inflammatory bowel diseases

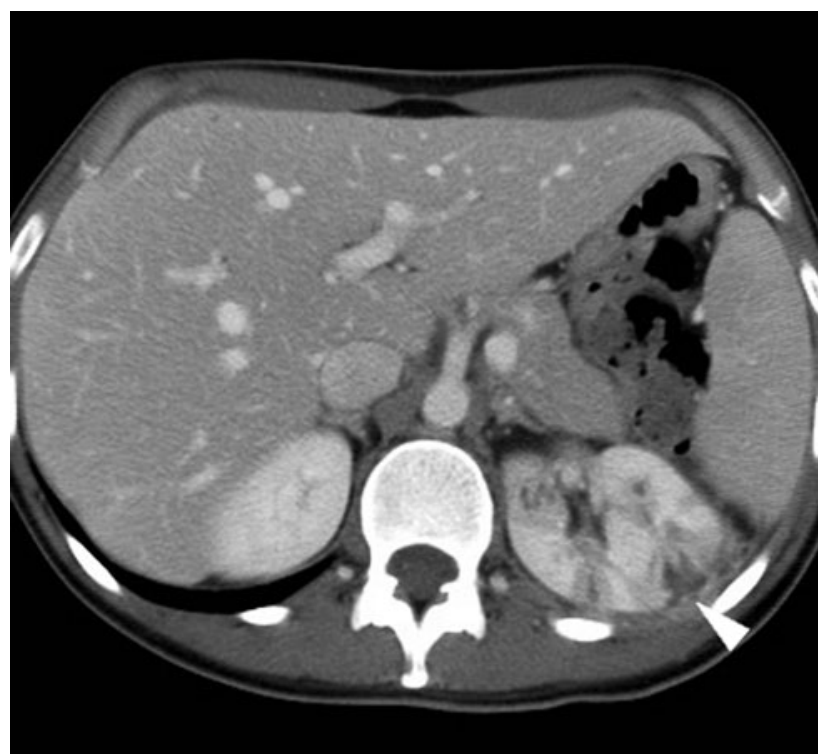

Fig. 1. 25-Year-old female with $C D$, fever, and flank pain. Contrast-enhanced CT image shows enlarged left kidney compared to contralateral one, with "striated nephrogram" appearance consistent with acute pyelonephritis complicated by a limited abscess collection (arrowhead).

frequently undergo imaging studies: the total effective dose from repeated CT examinations over a 5-year period has been estimated to be highest in CD patients, with some $(7 \%)$ of them exposed to very high radiation levels $(>50 \mathrm{mSv}$ ) [2,14]. Therefore, unenhanced CT to investigate urolithiasis and renal colic in CD patients $<50$ years of age should be prescribed with caution to limit their cumulative lifetime radiation exposure. Although with variable diagnostic accuracy, ultrasound screening for lithiasis and/or hydronephrosis may be preferable. Careful search for calcific stones is advised when interpreting contrast-enhanced small bowel CT studies.

\section{Urinary tract infection}

Very common especially in female CD patients, cystitis and simple urinary infections do not require imaging unless presentation is severe or complications are suspected. Characteristically manifesting with flank pain, fever and dysuria, pyelonephritis from ascending, untreated bacterial infection may result in renal damage. Although with limited sensitivity (20\%-25\%) when compared with CT, ultrasound is helpful as first-line diagnostic approach in patients with urinary infections. Sonographic appearances of pyelonephritis include renal enlargement with either focal, poorly marginated hypoechoic areas representing interstitial edema, or diffuse echogenicity changes with loss of corticomedullary differentiation and compressed, poorly visible renal sinus 

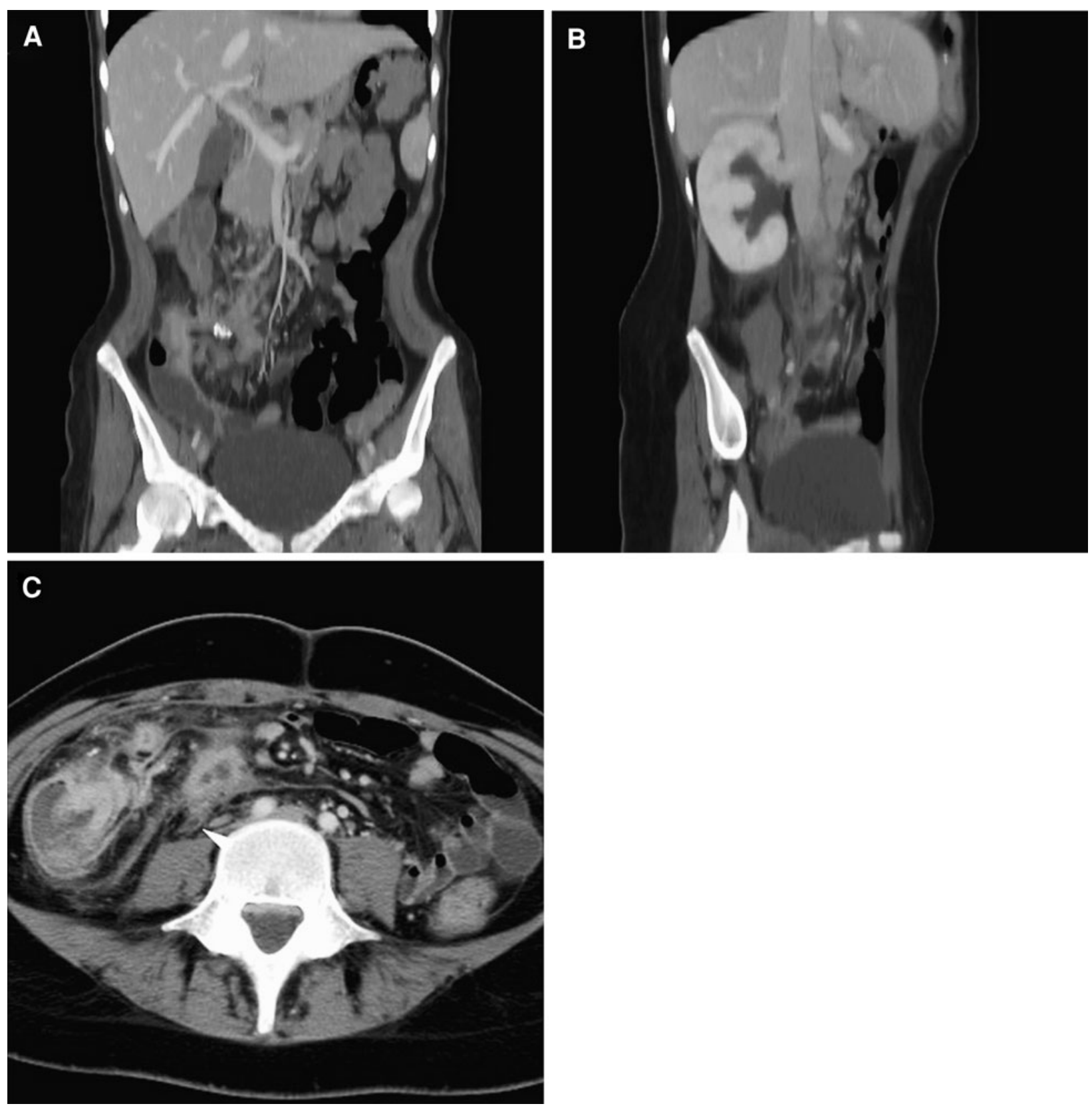

Fig. 2. 19-Year-old female with $C D$, previous segmental ileal resection 1 year earlier. Coronal- $(\mathbf{A})$, oblique- $(\mathbf{B})$ reformatted, and axial (C) images from urgent contrast-enhanced CT without bowel distension disclose recurrent ileocecal disease with con-

fat; abscesses may be identified as hypo-anechoic collections sometimes with mobile debris $[15,16]$.

Contrast-enhanced CT is the mainstay technique to image acute bacterial pyelonephritis. Features including variable renal enlargement, the "striated nephrogram" sign with multiple wedge-shaped hypovascular areas extending from the renal cortex to the papilla and associated findings such as Gerota's fascia thickening, perinephric fat stranding and fascial fluid can be occasionally

vergent enteric loops in the right iliac fossa indicating fistulization, plus ipsilateral hydronephrosis with preserved renal parenchymal thickness and enhancement caused by lumbar ureter encasement by the inflammatory mesenteric mass (arrowhead in $\mathbf{C}$ ).

identified in both urgent and elective studies in CD patients. Abscesses appear as intra- or extrarenal hypodense collections with peripheral rim enhancement (Fig. 1) $[15,16]$.

Considering the above-discussed concern for CTrelated radiation exposure, gadolinium-enhanced MRI is becoming increasingly established as a highly accurate alternative modality to image acute pyelonephritis and its complications. In young patients with $\mathrm{CD}$, the lack of 


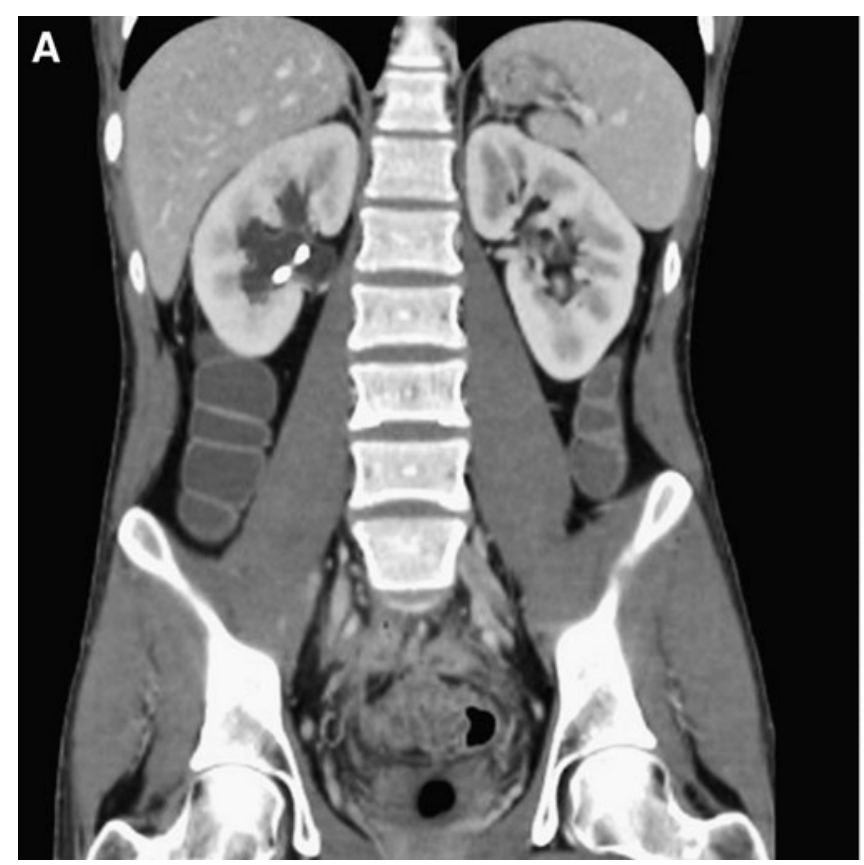

Fig. 3. 21-Year-old male patient with fistulizing CD, without urinary symptoms. Multiplanar contrast-enhanced CT enterography shows right-sided hydronephrosis treated with

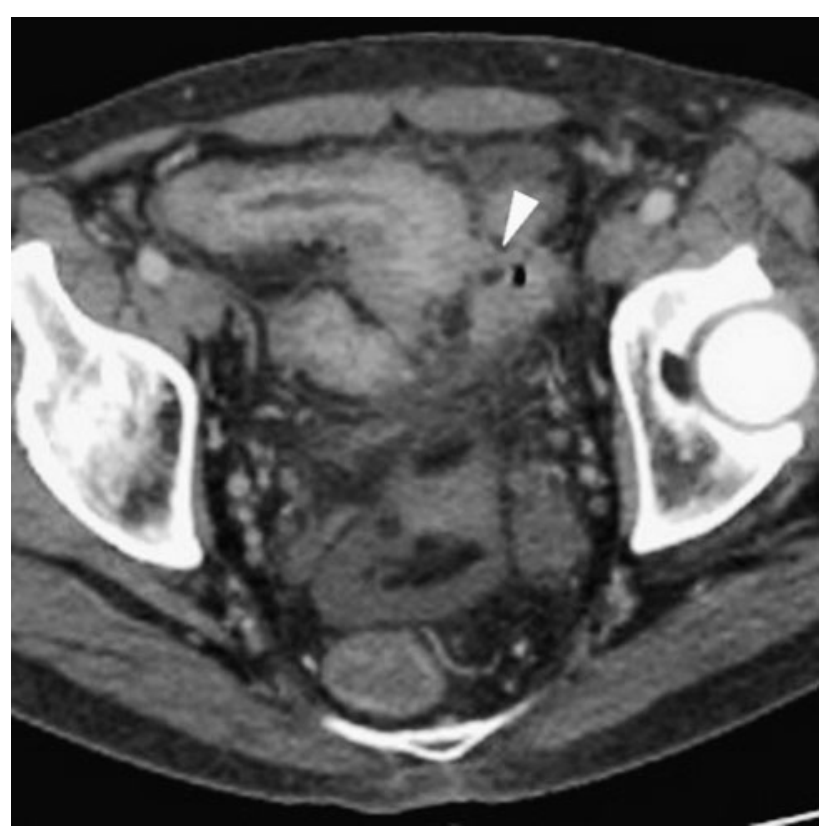

Fig. 4. 53-Year-old male with medically treated CD, complaining of episodical pneumaturia and fecaluria. Moderate leukocytosis and raised C-reactive protein. Urinalysis positive for leukocytes, negative cultures. Images from contrast-enhanced CT enterography show retracted, inflamed upper left bladder corner containing air, closely adherent and in communication with actively inflamed ileum and convergent thickened sigmoid (arrowhead). Surgery confirmed enterosigmoid-vesical fistulization and required ileocecal resection, segmental sigmoid resection, and bladder repair.

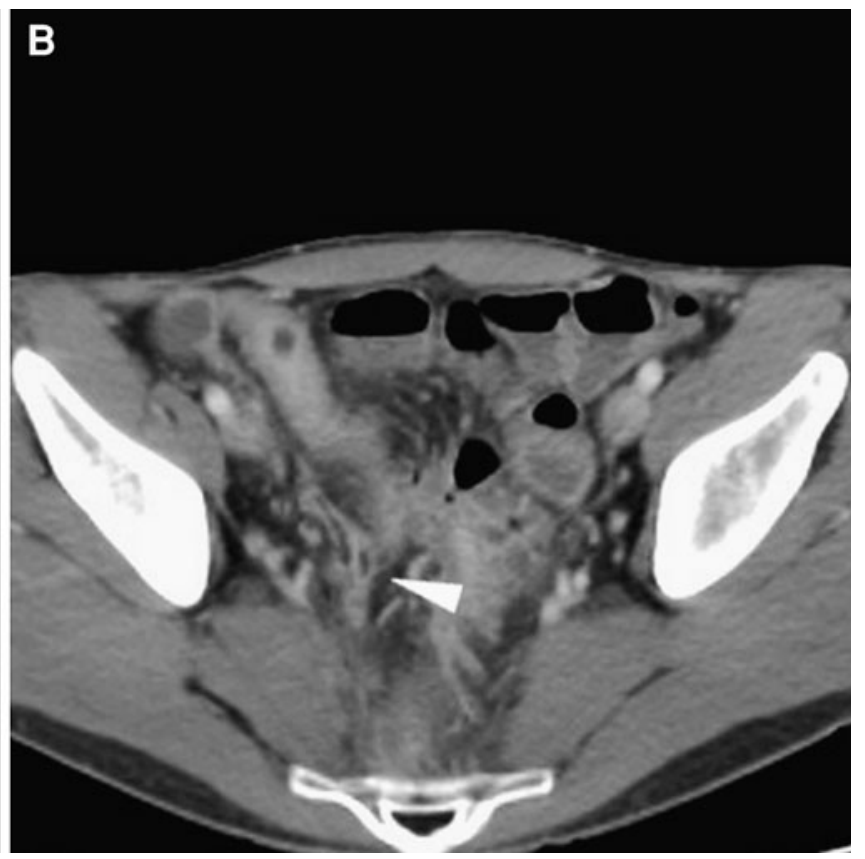

ureteral stent positioning (A). In the pelvis (B) active fistulizing enteric disease (arrowhead) causes upstream ureter dilatation.

ionizing radiation and the safety profile of paramagnetic contrast agents suggests use of MRI when pyelonephritis is suspected or during follow-up [15, 17].

\section{Obstructive uropathy}

A not-so-uncommon extraintestinal CD manifestation, urinary tract obstruction has been reported in 1.9\%-6\% of patients in the largest series. Often asymptomatic or underestimated in patients with active inflammatory bowel disease, hydronephrosis should be promptly diagnosed and treated considering the young age of most patients and the possible long-term renal function damage [18-20].

Almost invariably occurring on the right side, hydroureteronephrosis is caused by transmural bowel inflammation through ureter compression, fibrosis or encasement and is strongly associated with fistulizing ileocolic disease and inflammatory masses (Figs. 2, 3) [7, 18, 19].

In the past, hydronephrosis and other urologic disorders in CD patients were assessed with renal scintigraphy or intravenous pyelogram, probably resulting in underestimation of abnormalities [18, 21]. Currently, collecting systems dilatation may be detected with ultrasound and is further investigated by contrast-enhanced CT or MRI. Imaging features include the association of actively enhancing, thickened bowel segments with upstream ureteropelvic dilatation (Figs. 2, 3).

Right-sided hydronephrosis should be carefully sought for during cross-sectional CD imaging as it may 


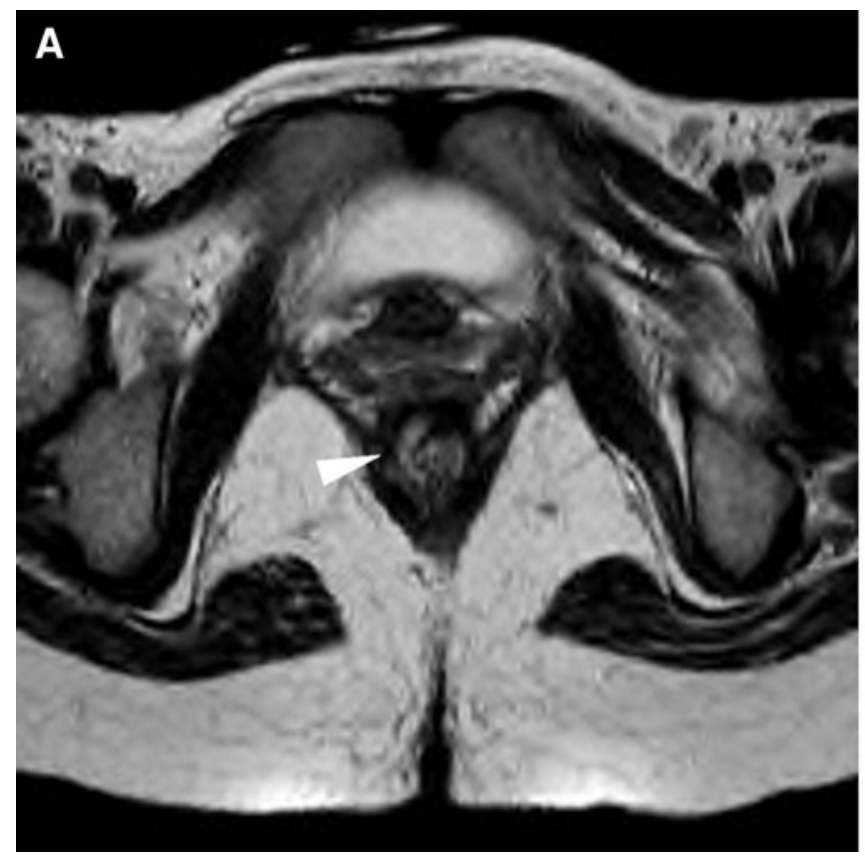

Fig. 5. 36-Year-old woman with history of CD, clinical suspicion of anovaginal fistula. Axial T2-weighted image from unenhanced MRI (A) confirms ventral left paramedian anal

lead to altered surgical management. Renal parenchymal thickness and functional status should be reported to facilitate treatment choice. Alternative causes of collecting systems dilatation include urolithiasis, chronic urine retention, and ureteropelvic junction obstruction [18].

Most patients are treated surgically with ileocolic resection plus ureterolysis, preoperatively positioned double-J stent minimizes the risk of ureteral damage during surgery (Fig. 3) [7, 8, 19].

\section{Enterovesical fistulization}

Urinary bladder communication from the adjacent diseased bowel represents another uncommon yet serious urologic $\mathrm{CD}$ complication, with a reported incidence of $2 \%-3.5 \%$. Very rare in females because of the presence of the uterus and adnexa, bladder fistulization results from penetration of full-thickness chronic ileal inflammation, whereas exceptional cases involve the colon or rectum. Notably, CD represents the most frequent cause of enterovesical fistulas, particularly in young patients [7, 11, 22, 23].

Suggestive clinical manifestations include pneumaturia and fecaluria, along with pain, pyuria, and hematuria; alternatively, patients with long-standing CD complain of recurrent urinary infections. Enteric bacteria such as Escherichia coli are the usual infective agents [22, 24, 25]. Oral or rectal administration of indocyanine green dye or other agents represents an easy, cheap, and highly accurate investigation to confirm the presence of vesical fistulization [26].

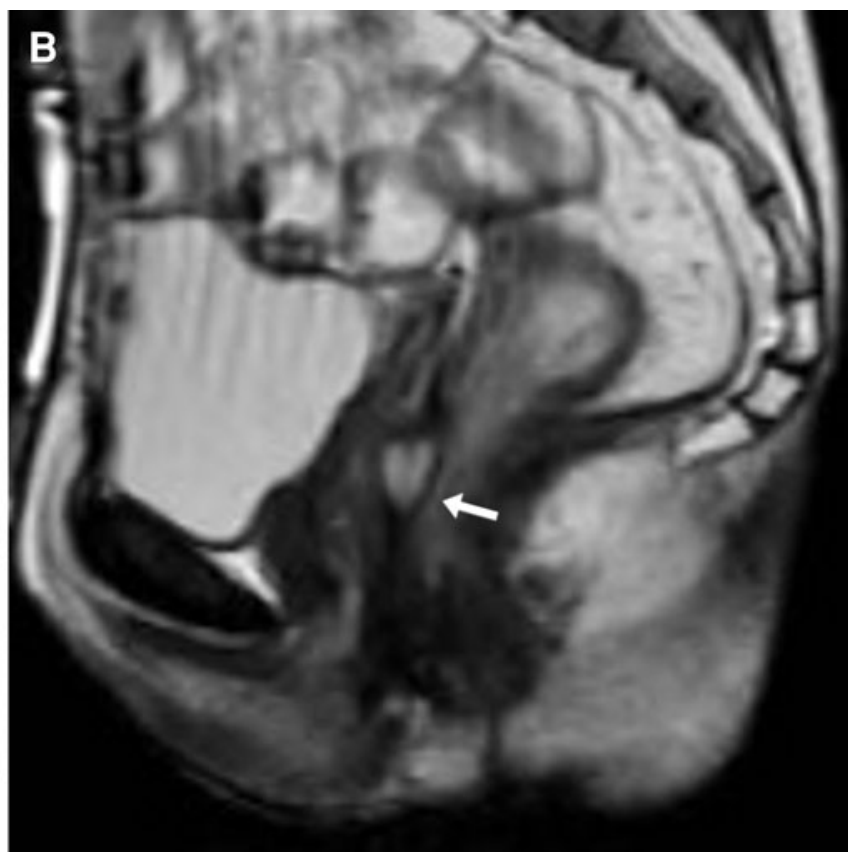

fistula (arrowhead), giving rise to fluid-filled abscess collection occupying the anovaginal septum, as seen on sagittal T2weighted image (arrow in B).

Although cystoscopy may identify the fistulous opening, past series reported limited accuracy for both radiological and endoscopic preoperative detection of enterovesical fistulas. Rarely, fistulas may be directly visualized when opacified by orally administered contrast, during fluoroscopic small bowel studies, intravenous urography or cystography $[8,22,23,25]$.

As most CD patients are currently investigated with contrast-enhanced CT or MRI, when interpreting elective and urgent cross-sectional studies radiologists should pay attention to features consistent with vesical fistulization, such as focal abnormalities or retraction of the bladder wall, adhesion and tethering of thickened adjacent bowel loops. Air in the bladder lumen is the most usual although indirect finding (Fig. 4) [23].

Refractory to conservative treatment, enterovesical CD-related fistulas need surgery to eradicate chronic urinary infection and prevent systemic sepsis. Affected bowel resection, sometimes with temporary ileostomy, includes bladder defect excision and closure [22, 24, 25].

\section{Genital complications}

Disorders involving the reproductive tract are common in $\mathrm{CD}$ patients, particularly in females. Sometimes gynecological complaints precede the diagnosis of the chronic inflammatory bowel disease, therefore persistent, unexplained genital abnormalities should raise suspicion of underlying CD [27, 28]. Direct extension of fistulizing transmural intestinal disease to the pelvic organs is the 

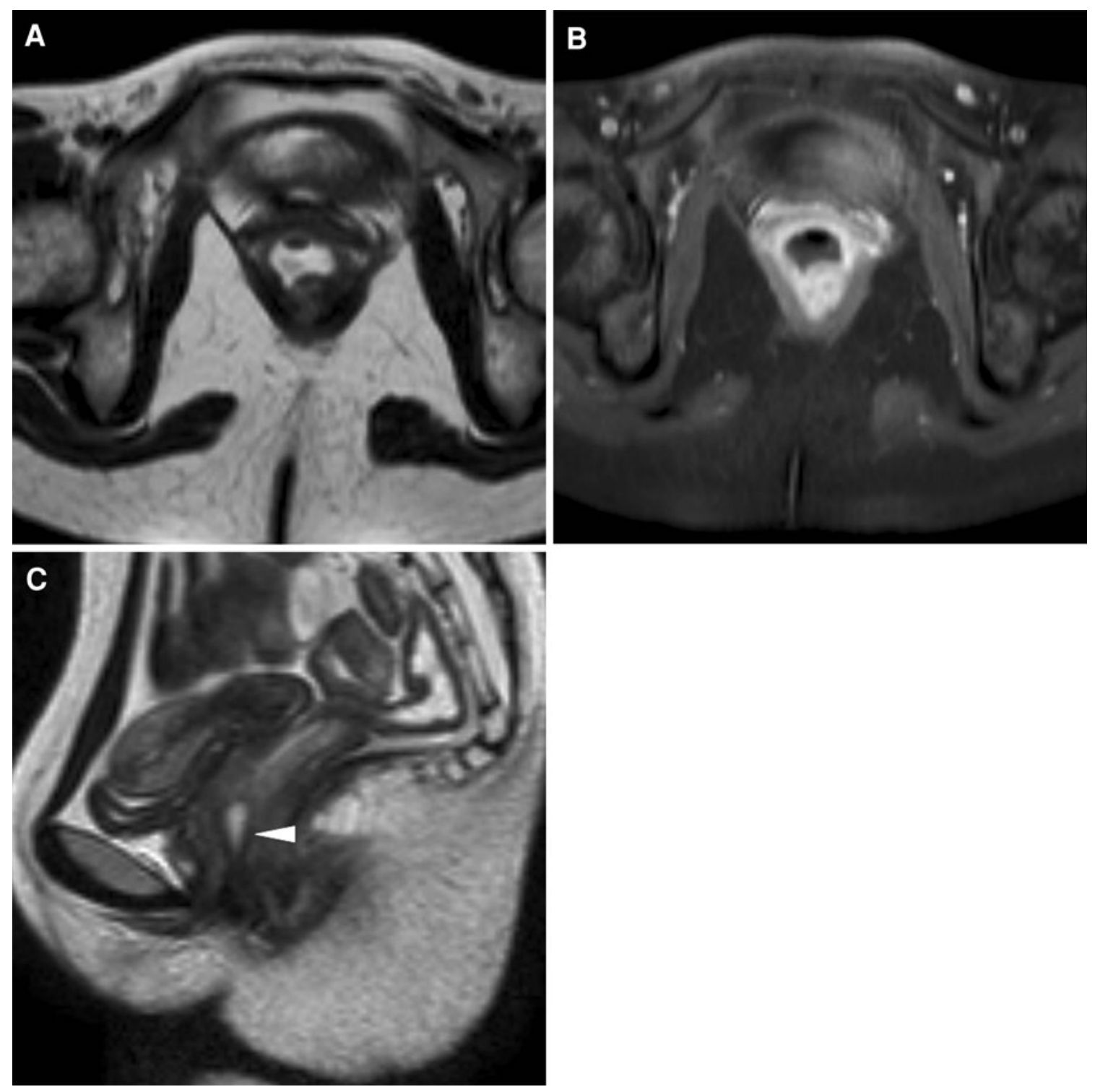

Fig. 6. 27-Year-old female with CD. Anovaginal septum abscess is detected by MRI as a fluid-filled cavity with nondependent gas bubble on axial T2-weighted image (A), showing strong peripheral enhancement on axial fat-suppressed (B) postcontrast T1-weighted image. After surgical

usual mechanism, whereas rarely "metastatic" CD occurs in the perineum and external genitalia separated from the bowel. Furthermore, systemic effects of chronic disease including malabsorption and malnutrition often cause altered fertility and menstrual cycle, impaired sexuality and psychosocial dysfunction $[9,10]$.

\section{Vaginal fistulas}

Representing the vast majority (nearly 75\%) of genital complications, ano- and rectovaginal fistulas occur in up to $5 \%-10 \%$ of female CD patients, particularly with colonic and perianal disease, and may significantly impair

drainage and medical treatment, follow-up MRI shows significant size reduction of the abscess as seen on sagittal T2weighted image $(\mathbf{C})$, with arrowhead pointing to the smaller residual fluid collection.

their quality of life. Anovaginal fistulas are differentiated from rectovaginal ones according to the location of the internal orifice in the anal canal or above the anal sphincter. Although some cases are asymptomatic, characteristic manifestations including air, enteric, or fecal vaginal discharge with recurrent genital infections, usually allow an easy clinical diagnosis. Vaginal fistulas may respond to medical treatment with biologics, alternatively surgical resection of the involved bowel segments with advancement flap and/or diverting ileostomy is performed $[9,10]$.

After clinical assessment including surgical examination under anesthesia, vaginal fistulas may be visualized 


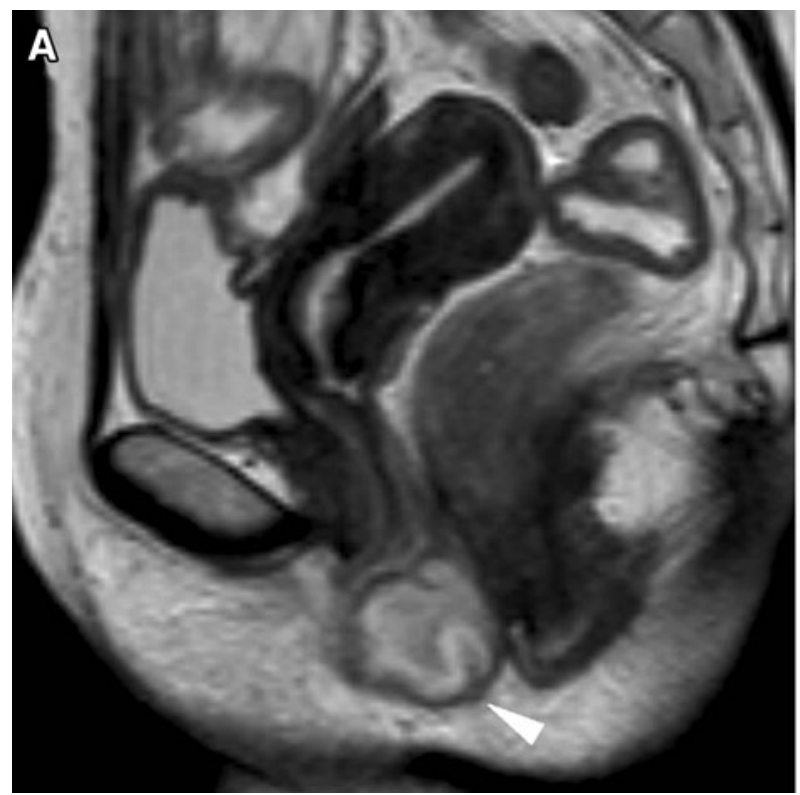

Fig. 7. 24-Year-old female with $C D$ and vulvar-perineal swelling, without appreciable fistulous orifices. Sagittal (A) T2weighted and axial fat-suppressed postcontrast T1-weighted (B) images from perianal MRI disclose a $3.5-\mathrm{cm}$ subcutane-

using fistulography, transrectal and transperineal ultrasound, or water-soluble contrast rectal enema [5, 10, 29].

Contrast-enhanced MRI provides a non-invasive, comprehensive visualization of perianal CD inflammatory abnormalities with their relationship with the anal sphincter complex. Classification of fistulas and abscesses according to anatomical Parks' and imaging-based St. James' Hospital staging schemes allows correct choice and planning of medical and surgical treatments. Simple vaginal fistulas, consisting in thin fluid-filled tracks lined by granulation tissue, may be identified as T2-hyperintense linear structures originating from the ventral aspect of the anal canal (Fig. 5). Positive contrast enhancement indicates inflammatory activity, although in our large, unpublished experience MRI has a somewhat limited (50\% sensitivity) accuracy for simple anovaginal fistulas. Conversely, abscess collections occupying the anovaginal septum are very easily detected as fluid-like, nonenhancing collections, with peripheral rim enhancement after intravenous contrast (Fig. 6). Furthermore, reproducible MRI studies allow confident reassessment of perianal inflammatory abnormalities following treatment (Fig. 6) [3-6].

\section{Internal genital fistulas}

Very uncommonly, transmural CD inflammation of the ileum, rectum, or proximal colon may penetrate into the uterus and adnexa. Literature records sporadic cases of CD-related ileo-salpingeal fistulas, usually diagnosed intraoperatively and treated with en bloc resection of the

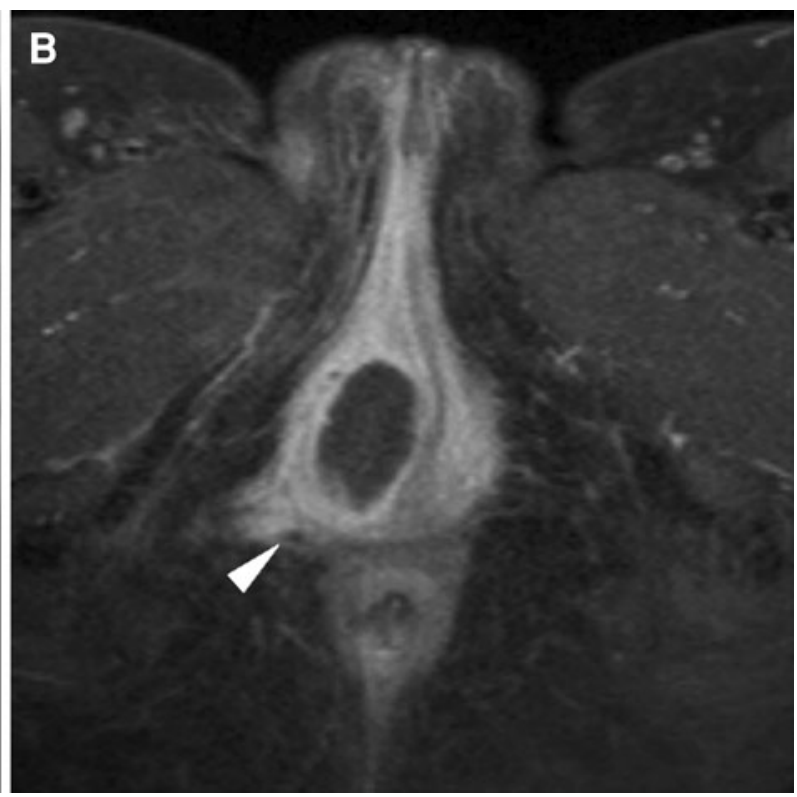

ous abscess collection in the right labial root (arrowheads) with non-enhancing fluid content and peripheral enhancement extending to the adjacent fat planes.

distal ileum with the affected fallopian tube [10, 30]. When interpreting cross-sectional imaging studies in CD patients, adnexal abnormalities particularly with abscesslike appearance should be reported and gynecological consultation suggested.

\section{Vulvar involvement}

Perineal and vulvar abnormalities are commonly encountered in female CD patients. In the setting of chronic inflammatory bowel disease, complaints of local discomfort, pain, or dyspareunia and physical findings such as labial swelling, erythema, tender deep ulcerations, nodular masses, or draining sinuses should be considered CD related. Otherwise, when intestinal disease is not already known, these nonspecific manifestations may be confused with a wide spectrum of conditions including Bartholini's gland cysts, hidradenitis suppurativa, granulomatous diseases (Behcet's and sarcoidosis), sexually transmitted infections (including syphilis, genital herpes, and lymphogranuloma venereum), or even with sexual abuse [10].

Vulvar CD most usually develops through direct extension from perianal and anovaginal inflammatory changes (Fig. 7). Conversely, "metastatic" CD refers to vulvar involvement noncontiguous to the gastrointestinal tract: reported in both adults and children, this very rare entity may precede or coexist with intestinal manifestations and represents a challenging diagnosis and a possibly unrecognized cause of vulvar pain [27, 28, 31, 32]. 

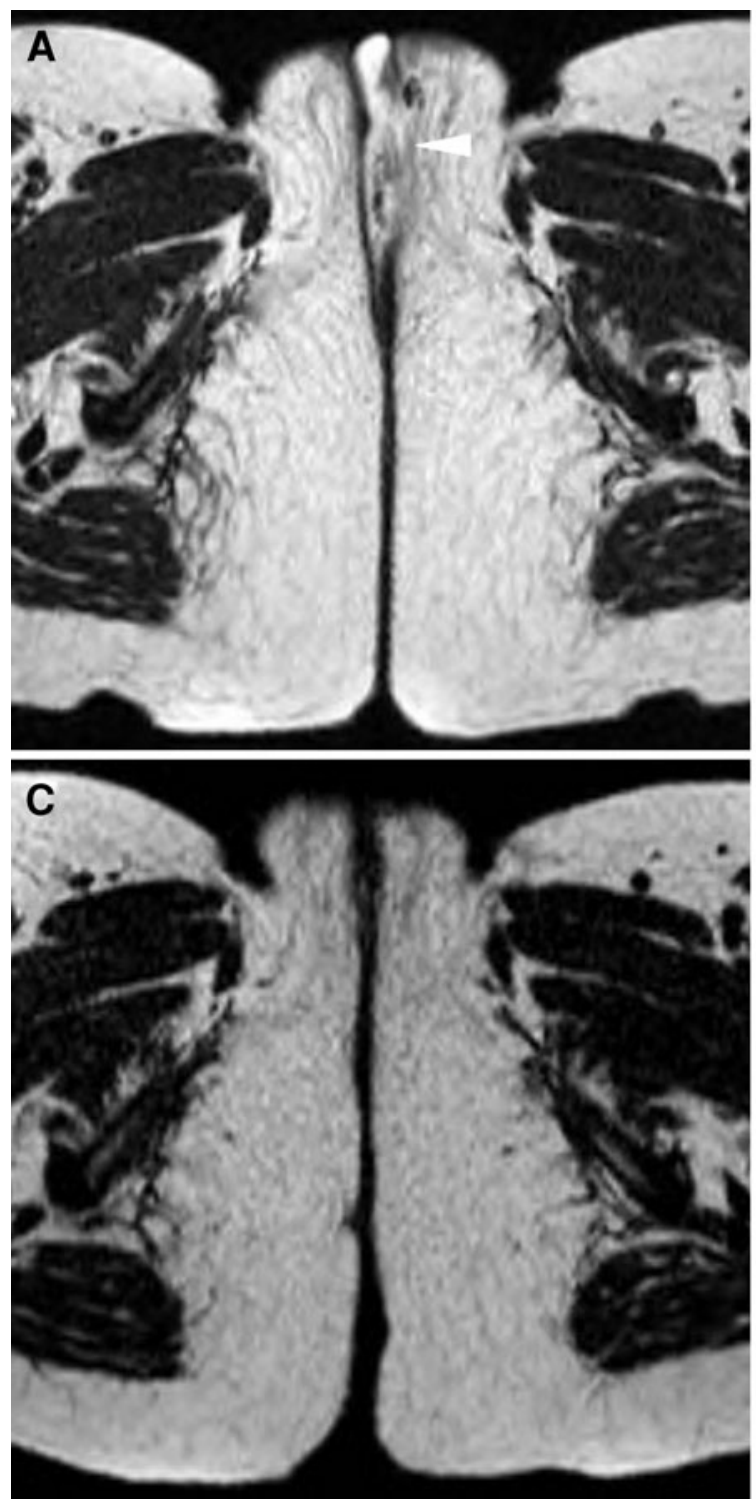

Fig. 8. 30-Year-old woman complaining of bloody diarrhea, fever, and abdominal pain for 1 month, with left-sided vulvar swelling, inflammation, and muco-hematic discharge. No abnormalities of the internal genital organs. MRI disclosed thickened left vulvar skin and subcutaneous tissue with T2signal hyperintensity (A) and positive contrast enhancement (B) consistent with inflammatory changes with small abscess

Inflammatory changes including vulvar skin and subcutaneous thickening with T2-weighted hyperintense signal and positive contrast enhancement, sometimes fistulas or abscess cavities are exquisitely depicted by MRI (Fig. 8), and should be sought for and reported when reviewing perianal or intestinal MRI studies in female patients with known or suspected CD [31, 33].

Long-term medical treatment with metronidazole alone or in combination with steroids is effective in treating clinical and imaging manifestations (Fig. 8), whereas advanced cases may require surgical vulvectomy $[28,33]$.

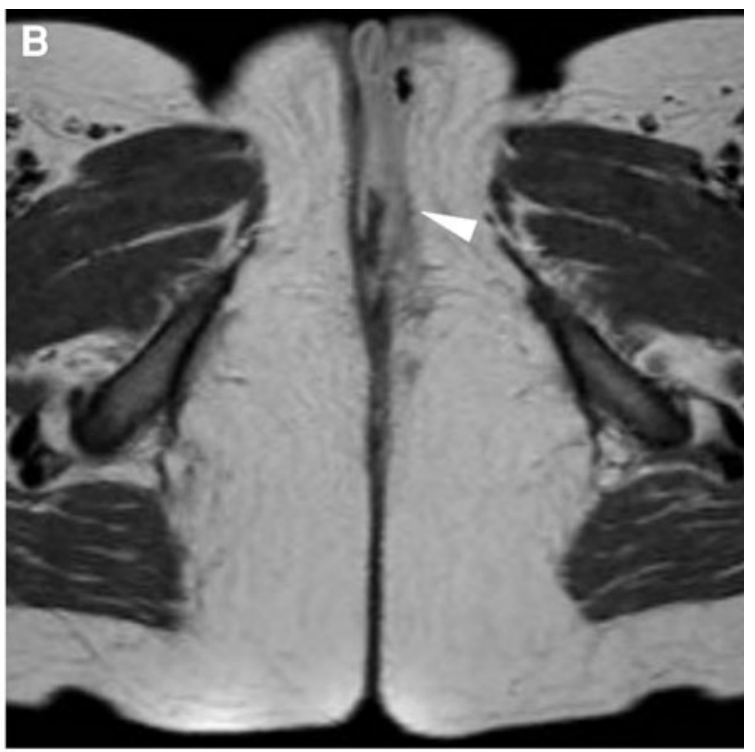

formation (arrowheads). Perianal and anovaginal inflammation was excluded at MRI, endoanal and transperineal US. Endoscopy allowed diagnosis of colonic CD. After systemic and enema steroid treatment, prompt clinical resolution of both intestinal complaints and vulvar involvement was confirmed by regression of MRI inflammatory changes (C).

\section{Male genital involvement}

Compared to females, male patients with $\mathrm{CD}$ rarely suffer from genital involvement. A few reports describe prostate gland and proximal urethra abscess formation from direct extension of perianal inflammatory disease. Clinical presentation includes local swelling or ulcerations, and pelvic-perianal MRI detects these abnormalities as fluid-filled, peripherally enhancing structures usually associated with perianal fistulas (Fig. 9) [10, 34, 35]. Furthermore, exceptional cases of metastatic CD as 


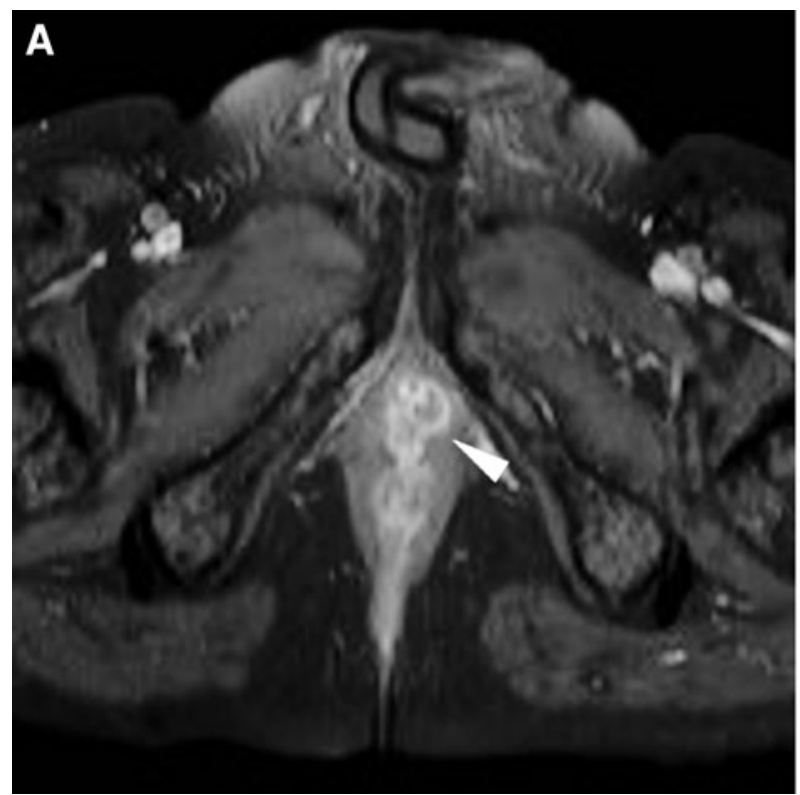

Fig. 9. In a 56-year-old male with longstanding CD, axial fatsuppressed (A) T1-weighted image after intravenous contrast discloses ventral anal fistula giving rise to periurethral abscess collection (arrowhead) located just caudally to the

solitary, non-healing ulcers or plaques involving the skin and subcutaneous planes of the penis and scrotum have been described [34].

\section{Conclusions}

Awareness of common and unusual complications involving the urinary tract and genital organs and knowledge of their imaging appearances is necessary to recognize and correctly report them when interpreting cross-sectional studies in patients with CD. Often clinically underestimated, these complications need urological and gynecological consultation to receive appropriate treatment [7-10].

\section{References}

1. Rimola J, Rodriguez S, Cabanas ML, et al. (2011) MRI of Crohn's disease: from imaging to pathology. Abdom Imaging. doi: 10.1007/s00261-011-9797-7.

2. Van Assche G, Dignass A, Reinisch W, et al. (2010) The second European evidence-based consensus on the diagnosis and management of Crohn's disease: special situations. J Crohns Colitis 4:63-101

3. Maccioni F, Colaiacomo MC, Stasolla A, et al. (2002) Value of MRI performed with phased-array coil in the diagnosis and preoperative classification of perianal and anal fistulas. Radiol Med 104:58-67

4. Halligan S, Stoker J (2006) Imaging of fistula in ano. Radiology 239:18-33

5. Szurowska E, Wypych J, Izycka-Swieszewska E (2007) Perianal fistulas in Crohn's disease: MRI diagnosis and surgical planning: MRI in fistulazing perianal Crohn's disease. Abdom Imaging 32:705-718

6. Torkzad MR, Karlbom U (2010) MRI for assessment of anal fistula. Insights Imaging 1:62-71

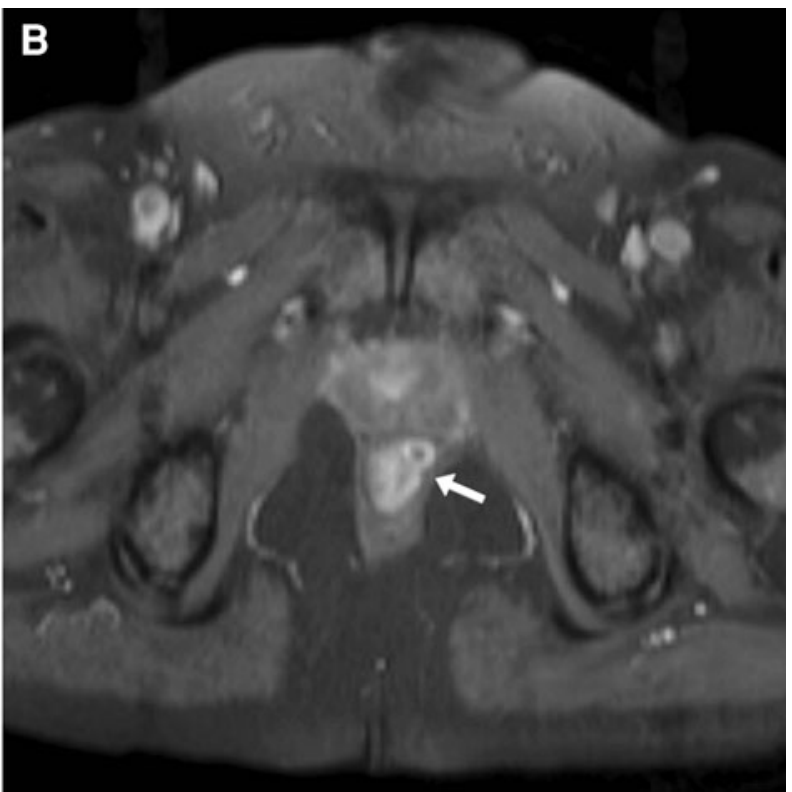

prostate gland. In a different patient, post contrast axial fatsuppressed T1-weighted image (B) shows retroprostatic abscess with peripheral enhancement (arrow), probably originating from a perianal sinus.

7. Ruffolo C, Angriman I, Scarpa M, et al. (2006) Urologic complications in Crohn's disease: suspicion criteria. Hepatogastroenterology 53:357-360

8. Ben-Ami H, Ginesin Y, Behar DM, et al. (2002) Diagnosis and treatment of urinary tract complications in Crohn's disease: an experience over 15 years. Can J Gastroenterol 16:225-229

9. De La Poza G, Lopez-Sanroman A, Taxonera C, et al. (2011) Genital fistulas in female Crohn's disease patients. Clinical characteristics and response to therapy. J Crohns Colitis. doi: 10.1016/j.crohns.2011.08.015.

10. Feller ER, Ribaudo S, Jackson ND (2001) Gynecologic aspects of Crohn's disease. Am Fam Physician 64:1725-1728

11. Sato S, Sasaki I, Naito H, et al. (1999) Management of urinary complications in Crohn's disease. Surg Today 29:713-717

12. Andersson H, Bosaeus I, Fasth S, et al. (1987) Cholelithiasis and urolithiasis in Crohn's disease. Scand J Gastroenterol 22:253-256

13. Eisner BH, McQuaid JW, Hyams E, et al. (2011) Nephrolithiasis: what surgeons need to know. AJR Am J Roentgenol 196:1274-1278

14. Kroeker KI, Lam S, Birchall I, et al. (2011) Patients with IBD are exposed to high levels of ionizing radiation through CT scan diagnostic imaging: a five-year study. J Clin Gastroenterol 45:34-39

15. Craig WD, Wagner BJ, Travis MD (2008) Pyelonephritis: radiologic-pathologic review. Radiographics 28:255-277

16. Stunell H, Buckley O, Feeney J, et al. (2007) Imaging of acute pyelonephritis in the adult. Eur Radiol 17:1820-1828

17. Martina MC, Campanino PP, Caraffo F, et al. (2010) Dynamic magnetic resonance imaging in acute pyelonephritis. Radiol Med 115:287-300

18. Ueno Y, Tanaka S, Kanao H, et al. (2006) A case of Crohn's disease with hydronephrosis caused by ureteropelvic junction obstruction. Eur J Gastroenterol Hepatol 18:1015-1018

19. Ben-Ami H, Lavy A, Behar DM, et al. (2000) Left hydronephrosis caused by Crohn disease successfully treated conservatively. Am J Med Sci 320:286-287

20. Siminovitch JM, Fazio VW (1980) Ureteral obstruction secondary to Crohn's disease: a need for ureterolysis? Am J Surg 139:95-98

21. Kruglik GD, Neiman HL, Sparberg M, et al. (1977) Urological complications of regional enteritis. Gastrointest Radiol 1:375-378

22. Solem CA, Loftus EV Jr, Tremaine WJ, et al. (2002) Fistulas to the urinary system in Crohn's disease: clinical features and outcomes. Am J Gastroenterol 97:2300-2305 
23. Wong-You-Cheong JJ, Woodward PJ, Manning MA, et al. (2006) From the archives of the AFIP: inflammatory and nonneoplastic bladder masses: radiologic-pathologic correlation. Radiographics 26:1847-1868

24. Yamamoto T, Keighley MR (2000) Enterovesical fistulas complicating Crohn's disease: clinicopathological features and management. Int J Colorectal Dis 15:211-215 (discussion 216-217)

25. McNamara MJ, Fazio VW, Lavery IC, et al. (1990) Surgical treatment of enterovesical fistulas in Crohn's disease. Dis Colon Rectum 33:271-276

26. Sou S, Yao T, Matsui T, et al. (1999) Preoperative detection of occult enterovesical fistulas in patients with Crohn's disease: efficacy of oral or rectal administration of indocyanine green solution. Dis Colon Rectum 42:266-270

27. Kingsland CR, Alderman B (1991) Crohn's disease of the vulva. J R Soc Med 84:236-237

28. Andreani SM, Ratnasingham K, Dang HH, et al. (2010) Crohn's disease of the vulva. Int J Surg 8:2-5
29. Maconi G, Ardizzone S, Greco S, et al. (2007) Transperineal ultrasound in the detection of perianal and rectovaginal fistulae in Crohn's disease. Am J Gastroenterol 102:2214-2219

30. Maun D, Vine A, Slater G (2006) Ileosalpingeal fistula: an unusual complication of Crohn's disease. Mt Sinai J Med 73:1115-1116

31. Pai D, Dillman JR, Mahani MG, et al. (2011) MRI of vulvar Crohn disease. Pediatr Radiol 41:537-541

32. Leu S, Sun PK, Collyer J, et al. (2009) Clinical spectrum of vulva metastatic Crohn's disease. Dig Dis Sci 54:1565-1571

33. Madnani NA, Desai D, Gandhi N, et al. (2011) Isolated Crohn's disease of the vulva. Indian J Dermatol Venereol Leprol 77:342-344

34. Reyes M, Borum M (2009) Severe case of genital and perianal cutaneous Crohn's disease. Inflamm Bowel Dis 15:1125-1126

35. Yamaguchi M, Kujiraoka Y, Saida Y, et al. (2001) Prostatic abscess in young males: a rare complication of Crohn's disease. Abdom Imaging 26:423-424 